Document downloaded from:

http://hdl.handle.net/10251/145752

This paper must be cited as:

De Battista, H.; Jamilis, M.; Garelli, F.; Picó, J. (03-2). Global stabilisation of continuous bioreactors: tools for analysis and design of feeding laws. Automatica. 89:340-348. https://doi.org/10.1016/j.automatica.2017.12.041

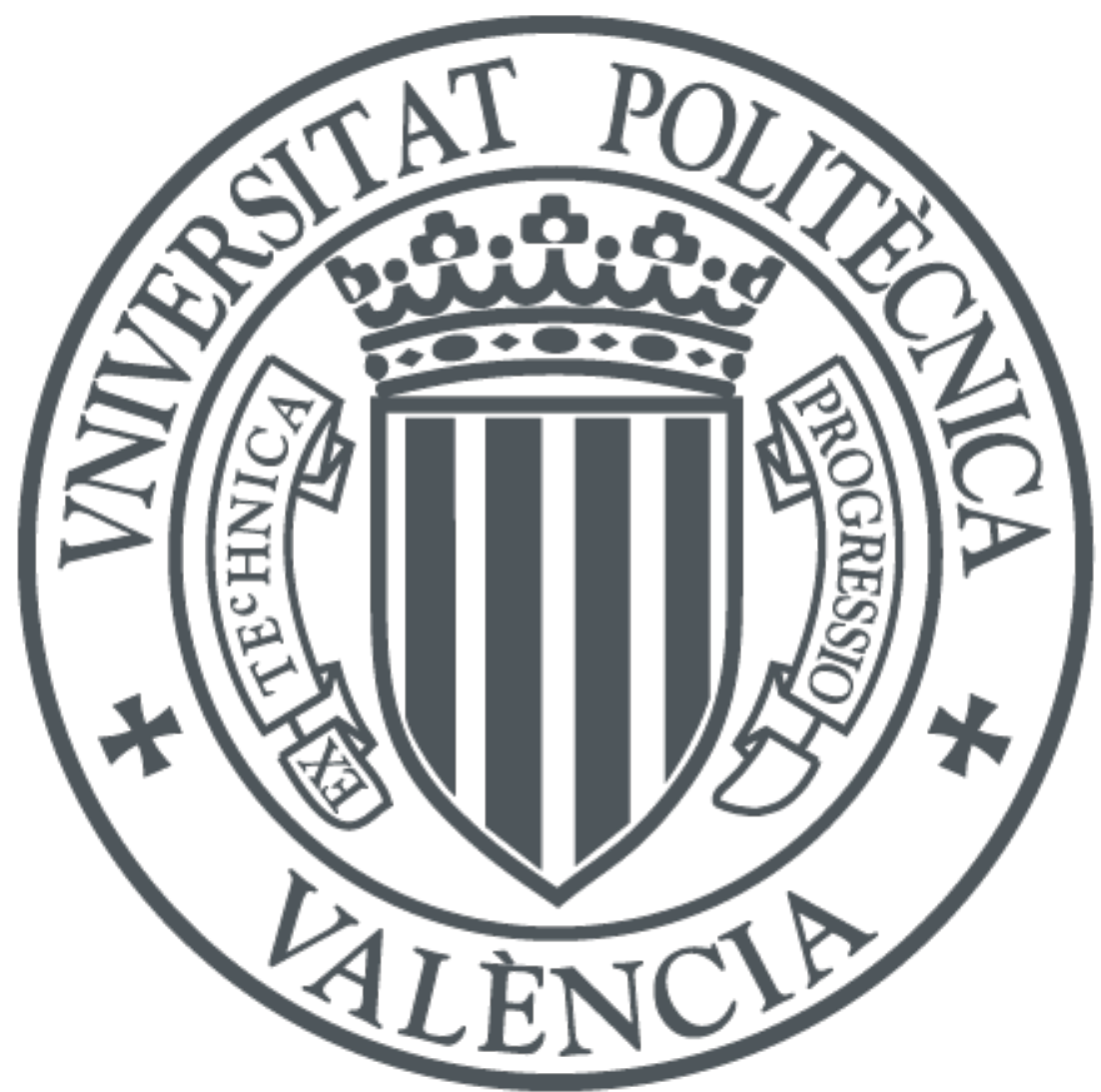

The final publication is available at

https://doi.org/10.1016/j.automatica.2017.12.041

Copyright Elsevier

Additional Information 


\title{
Global stabilisation of continuous bioreactors: tools for analysis and design of feeding laws
}

\author{
Hernán De Battista*, ${ }^{*}$ Martín Jamilis ${ }^{1}$, Fabricio Garelli ${ }^{1}$, Jesús Picó $^{2}$
}

\begin{abstract}
This work revisits the dynamic behaviour of stirred continuous reactors in which a single bioreaction with unknown kinetics occurs. Conditions on the feeding strategy to avoid washing out the biomass and falling in batch operation are obtained. These conditions derive in a closed positively invariant region including the desired operating point. It is stated that no closed orbits may exist in this region and, furthermore, that no fixed point exists but on one of its borders. Therefore, global stability is achieved by finding a feeding law that fulfills the aforementioned invariant conditions and gives a single equilibrium for a first-order dynamics. These results are useful to determine the stability properties of different control laws and, more importantly, to design new ones. Differing from previous results, the main advantages of the proposed approach are its simplicity and that input saturation does not affect stability results. The potentiality of the developed tools is illustrated by means of classical and novel feeding laws.
\end{abstract}

Key words: Bioprocess control; nonlinear control; invariant control.

\section{Introduction}

A continuous bioreactor is a vessel where a biochemical reaction takes place while fresh medium is continuously supplied and an effluent stream is withdrawn to keep volume constant. We focus on continuous stirred-tank bioreactors in which biomass is suspended in the liquid medium and the composition of the effluent is supposed to be the same as in the vessel.

This type of bioreactors has been widely used in industry during the last decades with several purposes: either to produce chemical compounds, to cultivate biomass, for extraction of intracellular products and in bioremediation.

They are also receiving a renewed interest in research. Since microbial growth occurs in an unchanging environment, continuous bioreactors are a source for large volumes of uniform cells or protein. This is fundamental for low noise characterisation of engineered microorganisms and biological circuits in synthetic biology [5, 26, 28].

Continuous bioreactors are long term processes along which set-points, control objectives, dynamic behaviour and environmental conditions may change. For instance, in research applications it is interesting to grow cultures under different conditions in order to properly characterise the microorganisms and find optimum productivity

\footnotetext{
*Corresponding author. Tel. $(+54) 221425$ 9306, E-mail: deba@ing.unlp.edu.ar.

${ }^{1}$ Grupo de Control Aplicado (GCA), Instituto LEICI, UNLPCONICET. Facultad de Ingeniería, Universidad Nacional de La Plata, Argentina.

${ }^{2}$ Institut d'Automàtica i Informàtica Industrial, Universitat Politècnica de València, Spain.
}

$[20,31]$.Therefore, global stability is essential for their successful control. To make most profit of the process, it is also convenient to minimise the transient between consecutive steady state operating points.

The control of continuous bioprocesses have been addressed using different design tools. Linear control theory has been applied in [11]. Exact feedback linearisation has been exploited, for instance, in [2, 21, 23], but input flow saturation impacts directly on the controller stability and performance. Lyapunov theory has been also used both for stability analysis and control design [27, 18, 17]. In [27], Lyapunov functions are used to determine domains of attraction of stable equilibria for open-loop processes in which the dilution rate is constant. In [18], sampled measurement of substrate concentration is used for feedback control but requires a model of the growth kinetics. In [14], the control of an age-structured continuous bioreactor modelled by a partial differential equation is addressed, but the effect of substrate on growth rate is not considered. In [17] globally stable feedback laws are designed, being the feedback gain dynamically adapted in such a way that control never saturates. In this control, speed of convergence is not an issue, so it is not set by controller tuning. There are other global control approaches [24], but they often do not explicitly consider the constraints on the input flow.

It is proposed here a simple and systematic methodology to design globally stabilising controllers for continuous bioreactors involving a pure culture growing on one limiting substrate. The trade-off between convergence speed and global stability becomes clear. One of the main advantages of the proposed approach is that input flow saturation does not affect stability, so the designer should not 
care about it. From a bioengineering viewpoint, this paper offers a mathematical analysis to re-think and understand trade-offs and limitations of empirical feeding laws.

As in previous papers in the literature $[2,17]$, we derive our results from a mass balance model of a continuous bioprocess in which a single bioreaction occurs. Since no particular expression for the reaction kinetics is considered, this model is suitable to describe the growth of a broad variety of microorganisms. The method presented here is also a basis for analysing and designing controllers for more complex bioreactions.

\section{Operating modes and dynamic model}

\subsection{Modes of operation}

In industry, continuous reactors are often operated as chemostats, that is the pump feeds fresh medium into the vessel at a constant rate. Chemostats reach their steady state when the dilution of the culture equals the microbial growth. Thus, the experimenter manipulates the specific growth rate of microorganisms by setting the set-point of the feeding pump. Chemostats suffer from some limitations, however. In fact, they are not reliable to regulate specific growth rates close to maximum since an unexpected or unmodelled growth inhibition may lead to biomass wash-out. Additionally, the culture might be lost during transient from one steady state to another one unless set-point changes are made slow enough. Finally, multiplicity occurs when, as usual, the growth is inhibited by a nutrient in excess.

As alternatives to chemostats, different closed-loop strategies have been developed. For instance, nutristats regulate substrate concentration at a given set-point. This operating mode avoids multiplicity and allows driving the biochemical reaction to maximum specific growth rate conditions. This operation is restricted to those processes in which the nutrient can be reliably measured on-line in low concentrations like in $[15,25]$. On the other hand, turbidostats regulate cell density at a prescribed value $[16,8]$. Cell density is continuously monitored using a spectrophotometer/turbidometer to measure the optical density for control purposes [4], or other methods based on dielectric permittivity [10]. Other operating methods use on-line measurement of other variables such as $\mathrm{pH}$, dissolved oxygen (DO), oxygen uptake rate (OUR), oxygen transfer rate (OTR), chemical oxygen demand (COD) to indirectly regulate a key variable of the biochemical reaction [19, 29].

In all these modes of operation, the dilution rate is manipulated to drive the process towards the desired operating point. Typically, set-point step and ramp changes are implemented to study the effects of the specific growth rate on the production rate and other issues. However, arbitrary set-points for both biomass and substrate concentrations cannot be achieved by only manipulating the dilution rate because of reaction constraints. To overcome them, a piece-wise constant inlet substrate concentration profile is often implemented, limited by maximal biomass concentration or OTR constraints. The switching times are chosen separated enough, so that the process is mostly operated in steady-state. A time of five generations of microorganisms in the new macroscopical steady-state is considered enough to assure internal steady-state in the cell metabolism.

\subsection{Mass balance dynamic model}

In this paper, we consider continuous bioprocesses in which a single species of microorganisms grows in a perfectly stirred vessel. It is assumed that the growth is limited by a single carbon and energy source (CES) whereas other required nutrients are in excess or suitably regulated. It is important to remark that no particular model for the kinetics of the reaction is considered. In fact, bioreactions obeying different types of kinetics are comprised: monotonic kinetics like Monod, Teissier and Moser, inhibitory kinetics by excessive substrate like Haldane, inhibitory kinetics by excessive biomass like Contois, etc. [3]. Therefore, the results presented below apply to a very wide range of bioprocesses. Further, the extension to even more general processes involving dual substrates will be briefly discussed too.

Let us consider the bioreaction mass balance model:

$$
\begin{array}{rlrl}
\dot{X} & =\mu X-D(t) X & X & \in \Re_{+} \\
\dot{S} & =-y \mu X+D(t)\left(S^{i n}(t)-S\right) & S & \in \Re_{+}
\end{array}
$$

where $X$ and $S$ are the biomass and substrate concentrations in the reactor, respectively, $\mu$ is the specific growth rate, $D$ is the manipulated dilution rate, $y$ is the substrateto-biomass yield and $S^{i n}(t)$ is the substrate concentration in the inlet flow. In the mass balance model (1), endogenous metabolism and cell maintenance are neglected.

Mass balance model (1) fulfills the dynamic restriction

$$
\dot{Z}=D\left(S^{i n}(t)-Z\right)
$$

where $Z=y X+S$ is the amount of CES per volume that was supplied into the reactor and is currently part of the cells or diluted in the liquid medium.

Assumption 2.1. Let $\mu(X, S, q)$ be a globally Lipschitz function satisfying $\mu(X, 0, \cdot) \equiv 0, \quad \mu(X, S, \cdot)>0 \forall S>0$

where $q \in \mathcal{Q}$ gathers uncertain parameters and other variables ( $D O$, temperature, $p H$, etc.) affecting the kinetics.

Note that assumption 2.1 is not restrictive from the bioprocess viewpoint since it is verified by most of the kinetic models used in biotechnology.

Assumption 2.2. Let $S^{i n}(t)$ be positive and piece-wise constant. That is, given a set of instants $\left\{t_{j}\right\}$ that partitions the process time into intervals $I_{j}=\left[t_{j}, t_{j+1}\right)$ and a set of positive constants $\left\{S_{j}^{i n}\right\}$, then $S^{i n}(t)=S_{j}^{i n} \forall t \in I_{j}$. 
Recall that the metabolic steady-state is achieved some time after the transient in the reactor media vanishes. Therefore, time intervals $I_{j}$ are supposed to be much longer than the settling time of the closed-loop responses.

Assumption 2.3. The dilution rate is nonnegative and bounded $0 \leq D(t) \leq \bar{D} \forall t$.

In continuous mode, $D(t) \geq d \forall t \geq 0$, for some sufficiently small $d>0$. Note however that this constraint can be conveniently relaxed as follows:

Definition 2.1. We will say that a bioprocess operates in continuous mode when, for each time interval $I_{j}$, there exists $0 \leq T_{C j}<<\left(t_{j+1}-t_{j}\right)$ such that $D(t) \geq d \forall t \geq$ $t_{j}+T_{C j}$, for some sufficiently small $d>0$.

This definition includes processes in which the control saturates at $D=0$ during some time periods along the transient response.

Let us normalise the concentrations w.r.t. the piece-wise constant inlet substrate concentration $S^{\text {in }}(t)$ :

$$
x:=\frac{y X}{S^{i n}} \quad s:=\frac{S}{S^{\text {in }}} \quad z:=\frac{Z}{S^{\text {in }}}
$$

After applying this normalisation and some abuse of notation, the mass balance model is rewritten as:

$$
\begin{aligned}
\dot{x} & =\mu(x, s, q) x-D(t) x & & x \in \Re_{+} \\
\dot{s} & =-\mu(x, s, q) x+D(t)(1-s) & & s \in \Re_{+} \\
\dot{z} & =D(t)(1-z) & & z \in \Re_{+}
\end{aligned}
$$

where the state jumps at the switching instants $t_{j}$ :

$$
\left(x\left(t_{j}\right), s\left(t_{j}\right), z\left(t_{j}\right)\right)=\frac{S_{j-1}^{i n}}{S_{j}^{i n}} \lim _{t \rightarrow t_{j}^{-}}(x(t), s(t), z(t))
$$

Note that (4) is a non-minimal realisation of (1) since the state lives on subspace $L=\{(x, s, z) \mid z=x+s\}$.

\section{Invariance, equilibria and stability of continu- ous bioprocesses}

\subsection{Invariance}

Under assumption 2.3 , the set $\left[0, \max _{t} S^{i n}(t)\right]$ is an invariant set for $Z$. Furthermore, since both $X$ and $S$ satisfy (2) and are nonnegative under assumptions 2.1-2.3, then they are bounded by $S, y X \in\left[0, \max _{t} S^{i n}(t)\right]$. Therefore,

$$
I R=\left\{(x, s, z) \in\left[0, \frac{\max _{j} S_{j}^{i n}}{\min _{j} S_{j}^{\text {in }}}\right]^{3} \cap L\right\}
$$

in the normalised state space is positively invariant. Furthermore, concentrations evolve in such a way that $z$ is piece-wise monotonous in IR, i.e. it is either nondecreasing or non-increasing for every time interval $I_{j}$.
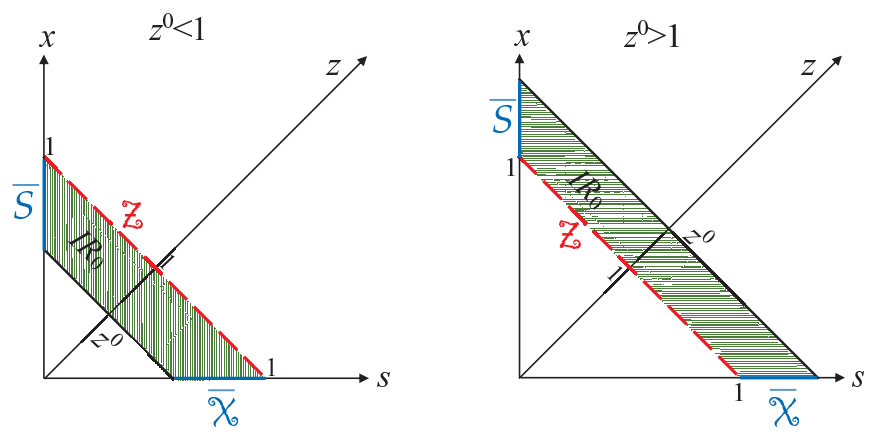

Figure 1: Invariant region $I R_{0}$ of (4) for initial conditions on $z^{0}<1$ (left) and $z^{0}>1$ (right). Invariant wash-out subregion $\overline{\mathcal{X}}$, equilibrium subregion $\mathcal{Z}$ and batch subregion $\overline{\mathcal{S}}$.

Since the time-dependent switching is supposed to occur at low frequency and the state jumps within the invariant region $I R$, it suffices to study the response of (4) for an arbitrary time period $I_{j}$ and an arbitrary initial condition in $I R$. Consequently, from now on we will omit subscript $j$ unless necessary.

Let us define the arbitrary initial state $P^{0}:=$ $\left(x^{0}, s^{0}, z^{0}\right) \in I R$. Then, the following set of $I R$ is also positively invariant:

$$
I R_{0}=\left\{(x, s, z) \in I R \mid z \in\left\langle z^{0}, 1\right\rangle\right\}
$$

where the notation $\left\langle z^{0}, 1\right\rangle:=\left[\min \left\{z^{0}, 1\right\}, \max \left\{z^{0}, 1\right\}\right]$ was used. By simple inspection of (4), invariant sets exist on $I R_{0}$ independently of the feeding law. They are:

$$
\begin{aligned}
& \overline{\mathcal{X}}=\left\{(x, s, z) \in I R_{0} \mid x=0\right\} \\
& \mathcal{Z}=\left\{(x, s, z) \in I R_{0} \mid z=1\right\}
\end{aligned}
$$

$\overline{\mathcal{X}}$ means biomass wash-out, whereas $\mathcal{Z}$ is the reaction invariant [30]. These invariant sets are depicted in Fig. 1.

\subsection{Equilibria}

Let us analyse first the equilibria of the autonomous system (4) with $D=0$, i.e. in batch operation mode. By simple inspection of (4) we realise that $z$ is constant in batch mode. Also, every point in $\overline{\mathcal{X}}$ is an unstable fixed point. Analogously, every point in

$$
\overline{\mathcal{S}}=\left\{(x, s, z) \in I R_{0} \mid s=0\right\}
$$

is a marginally stable fixed point. Furthermore, note all trajectories initiated in $I R_{0}$ with $x^{0}>0$ converge toward $\overline{\mathcal{S}}$ at constant $z$. Since $z$ is constant during the batch, no closed orbit exists on $I R_{0}$. Consequently all orbits not originated in a (non-isolated) fixed point of the autonomous system are straight lines pointing to $\overline{\mathcal{S}}$ at constant $z$. Note also that convergence to $\overline{\mathcal{S}}$ is asymptotic.

Let us see now possible equilibria of (4) in continuous operation mode. 
Assumption 3.1. Let the dilution rate be a globally Lipschitz time invariant feedback law $D(x, s)$ satisfying assumption 2.3.

Result 3.1. There are no closed orbits nor fixed points of system (4) in the interior of $I R_{0}$.

$I R_{0}$ is a closed positively invariant set. Since the system is planar on $L$ and $z$ is monotonous, neither strange attractors nor limit cycles can exist.

It is easy to see also that any fixed point is on the border of $I R_{0}$. On the one side, in continuous mode $(D>0)$, all orbits originated in $I R_{0}$, even those starting in $\overline{\mathcal{X}}$ and $\overline{\mathcal{S}}$, will converge to $\mathcal{Z}$. Since the system dynamics on $\mathcal{Z}$ can be described by a first-order differential equation, then only fixed points can exist on it. On the other, in batch mode $(D=0)$, all orbits originated in the interior of $I R_{0}$ will converge to a point in $\overline{\mathcal{S}}$ while $\overline{\mathcal{X}}$ and $\overline{\mathcal{S}}$ are sets of nonisolated equilibria. Therefore, also in batch mode, only fixed points on the border of $I R_{0}$ exists.

The desired operating point $P^{*}=\left(x^{*}, s^{*}, 1\right)$ of the process is, of course, on $\mathcal{Z}$, which with some abuse of notation can be written also as $P^{*}=\left(x^{*}, 1-x^{*}\right)$ or $P^{*}=\left(1-s^{*}, s^{*}\right)$.

\subsection{Stability of a continuous bioreactor}

Batch operation must be avoided, otherwise the state will not converge to $\mathcal{Z}$ but to $\overline{\mathcal{S}}$. In continuous mode, convergence to the wash-out equilibrium $P_{w}=(0,1,1)$ must be avoided. This point will be a stable node or a saddle, depending on the feedback control law. Of course, (semi-)global stabilisation of $P^{*}$ requires $P_{w}$ to be a saddle point attracting only the orbits originated in the invariant set $\overline{\mathcal{X}}$. However, this design specification is not enough in practice since unmodelled causes such as mortality can result in biomass was-out. It is therefore desired biomass concentration not to fall below too low concentrations.

In the following, we derive conditions for $\overline{\mathcal{X}}$ and $\overline{\mathcal{S}}$ to repel orbits originated in the interior of $I R_{0}$.

It is quite obvious from (4a) that if $D(\underline{x}, s) \leq$ $\mu(\underline{x}, s, q) \forall z \in\left\langle z^{0}, 1\right\rangle, \forall q \in \mathcal{Q}$, for some $\underline{x}<x^{*}$, then

$$
R_{X}=\left\{(x, s, z) \in I R_{0} \mid x \geq \underline{x}\right\}
$$

is positively invariant. If we accept $\underline{x}$ small enough, by continuity we simply have to check that $\overline{\mathcal{X}}$ is locally nonattractive.

Wash-out avoidance condition (WAC)

If the dilution rate verifies

$$
\lim _{x \rightarrow 0^{+}} D(x, s)-\mu(0, s, \cdot)<-\epsilon \quad \forall s \in\left\langle z^{0}, 1\right\rangle
$$

$\epsilon \geq 0$, then a positively invariant region $R_{X}$ exists for some sufficiently small $\underline{x}$. Note that a feeding law satisfying (11) avoids also convergence to the wash-out operating point $P_{w}$, i.e. makes $P_{w}$ a saddle point.
Strong batch avoidance condition ( $s B A C$ )

There are no equilibrium points on $\overline{\mathcal{S}}$ iff

$$
D(x, 0)>\epsilon \forall x \in\left\langle z^{0}, 1\right\rangle
$$

This condition can be very restrictive and, indeed, is not satisfied by some classical feeding laws. Assuming $s^{0}>0$, we can relax it by requiring $\overline{\mathcal{S}}$ to be repelling.

Weak batch avoidance condition (wBAC)

$\overline{\mathcal{S}}$ is locally non-attractive if $D(x, s)$ verifies

$$
\lim _{s \rightarrow 0^{+}} \frac{D(x, s)}{\mu(x, s, \cdot)}-x>\epsilon \quad \forall x \in\left\langle z^{0}, 1\right\rangle
$$

By continuity, if $D(x, s)$ fulfills (12) or (13), then there exists $\underline{s}$ sufficiently small such $D(x, \underline{s}) \geq \frac{x \mu(x, \underline{s}, q)}{1-\underline{s}} \forall z \in$ $\left\langle z^{0}, 1\right\rangle, \forall q \in \mathcal{Q}$, and, consequently the region

$$
R_{S}=\left\{(x, s, z) \in I R_{0} \mid s \geq \underline{s}\right\}
$$

is positively invariant. Let us call

$$
I R^{*}=R_{X} \cap R_{S} \subset I R_{0}
$$

Obviously, if the wash-out and batch invariance conditions hold, then $I R^{*}$ will be positively invariant.

Result 3.2. If $D(x, s)$ satisfies conditions (11), (13) and

$$
\left.\mu(x, s, q)\right|_{(x+s=1)}-\left.D(x, s)\right|_{(x+s=1)}=0
$$

has a single solution $\left(x^{*}, s^{*}\right) \in(0,1) \times(0,1)$, then all orbits originated in $I R^{*}$ globally converge to $P^{*}=\left(x^{*}, s^{*}, 1\right)$.

This result follows from the invariance of $I R^{*}$ and result 3.1. Since no equilibrium can exist in $I R^{*}$ outside $\mathcal{Z}$, it suffices to verify the existence of a single equilibrium on $I R^{*} \cap \mathcal{Z}$. In other words, it suffices to verify the existence of a single equilibrium for the first-order $x$-dynamics (4a) with $D(x, 1-x)$ or, analogously, for the $s$-dynamics $(4 \mathrm{~b})$ with $D(1-s, s)$. Note that all equilibria of the first-order $x$ - and $s$-dynamics on $\mathcal{Z}$ in $I R^{*} \cap \mathcal{Z}$ are also roots of (16). Recall that the wash-out equilibrium $P_{w}=(0,1,1)$, that is always solution of the first-order $x-$ and $s$-dynamics on $\mathcal{Z}$ for any bounded $D$, is outside $I R^{*}$. The same happens with the batch equilibrium $P_{b}=(1,0,1)$.

Remark: Result 3.2 applies to $I R^{*}=R_{X}$ when the sBAC (12) holds instead of the wBAC (13).

Note: $\epsilon=0$ in (11)-(13) gives nominal conditions for invariance of $I R^{*}$, whereas $\epsilon>0$ provides robustness against model uncertainties, delayed measurement and sampled control.

\subsection{Control design procedure}

Based on the previous results, we can summarise the procedure to design a globally stabilising feedback control law as follows. 
- Design $D(x, s)$ satisfying Assumption 3.1 that locally stabilises the desired operating point $P^{*}=\left(x^{*}, s^{*}\right)$.

- Shape $D(x, s)$ so that:

$$
\begin{aligned}
& \text { - the WAC (11) holds, } \\
& \text { - the BAC (13) or (12) holds, } \\
& \text { - (16) has a single solution }\left(x^{*}, s^{*}\right) \in(0,1) \times(0,1) \text {. }
\end{aligned}
$$

Recall that saturation of the control action $D$ does not affect global stability.

\section{4. (Semi-)global stability properties of locally sta- ble feedback laws}

We will illustrate now how the previous results can be used to determine the (semi-)global stability features of feeding laws, as well as to design (semi-)globally stable ones. We start our analysis with the classical open-loop control law to illustrate how the invariance conditions are to be checked and their relations with stability.

\subsection{Open-loop control (Chemostats)}

In chemostats, the dilution rate is fixed at the desired specific growth rate:

$$
D^{*}=\mu^{*}
$$

Chemostat dynamics have been widely studied from different approaches. Particularly, the Lyapunov-based results presented in [27] for open-loop bioreactors are very useful to check stability and domains of attraction in chemostats. The stability problem is addressed here but using the invariant tools developed in this paper. The purpose is not to find new results about chemostats stability but to adopt it as starting point to investigate stability of closed-loop controlled bioreactors. Additionally, some mathematical insight into chemostat limitations is given.

For $D^{*}=\mu^{*}$, the nominal strong batch avoidance condition (12) and the wash-out avoidance condition (11) become

$$
\begin{array}{ll}
s B A C: \mu^{*}>0 & \forall x \in\left\langle z^{0}, 1\right\rangle \\
W A C: \mu^{*}<\mu(0, s, \cdot) & \forall s \in\left\langle z^{0}, 1\right\rangle
\end{array}
$$

It is immediate to see that (18a) is fulfilled, so there are not equilibrium points on $\overline{\mathcal{S}}$. Conversely, the $(18 \mathrm{~b})$ is not always satisfied even for the simplest kinetics. In fact, depending on $\mu^{*}$ and the kinetic function, the biomass may be washed out or not.

The first-order dynamics on the reaction invariant $\mathcal{Z}$ can be obtained by evaluating the right hand side of $(4 \mathrm{a})$ or (4b) at $z=1$ and replacing $D(t)$ with $D^{*}$. For instance,

$$
\dot{s}=-\left(\mu(1-s, s, q)-\mu^{*}\right)(1-s)
$$

As predicted in Result 3.2, the substrate equilibria are $s=1$, that corresponds to $P_{w}$ and is outside $I R^{*}$, and the roots of (16). (a) kinetics

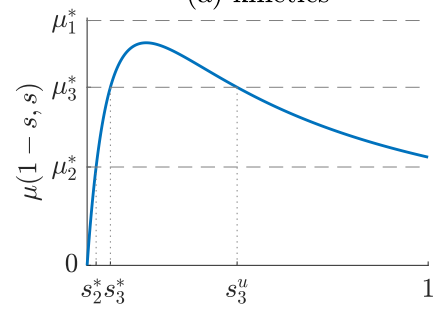

(c) $\mu_{2}^{*}<\mu(0,1)$

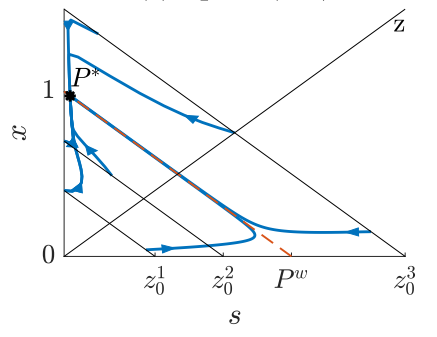

(b) $\mu_{1}^{*}>\mu_{\max }$

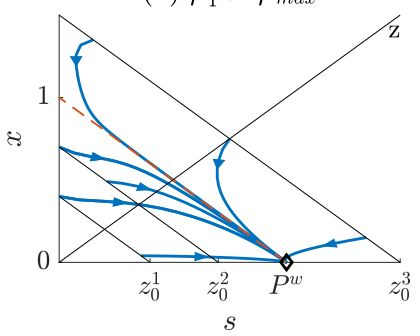

(d) $\mu(0,1)<\mu_{3}^{*}<\mu_{\max }$

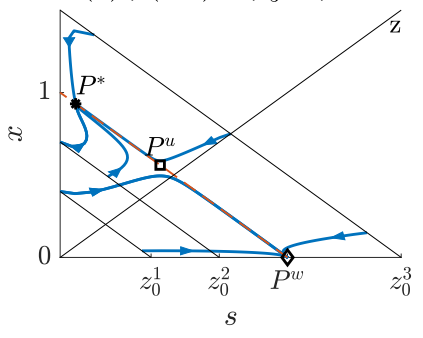

Figure 2: Chemostat, $D^{*}$ in eq. (17). (a) Specific growth rate $\mu(1-$ $s, s, q)$ and equilibria for different set-points $\mu^{*}$, (b-d) State portraits for different set-points; $*$ : set-point, $\diamond$ : undesired stable equilibrium, $\square$ : unstable equilibrium.

The kinetics with slight inhibition of Fig. $2 \mathrm{a}$ is very useful to illustrate the different behaviours observed in chemostats and how the developed tools apply. The state portraits for different set-points $\mu^{*}$ are showed in the remaining boxes of Fig. 2

The most undesired situation occurs when the maximum specific growth rate is overestimated (as $\mu_{1}^{*}$ in Fig. 2a). This happens when neglecting some limiting effect such as substrate inhibition, low DO concentration and $\mathrm{pH}$ out of range. In this case (Fig. $2 \mathrm{~b}$ ), the WAC (18b) is violated all along $s \in\left\langle z^{0}, 1\right\rangle$. It can be seen that (19) presents a globally stable equilibrium at $P_{w}$. On the other hand, the most favourable situation is when (19) exhibits a single equilibrium in the interior of $\mathcal{Z}\left(s_{2}^{*}\right.$ for the set-point $\left.\mu_{2}^{*}\right)$. Some illustrative state trajectories from different $z^{0}$ are plotted in Fig. 2c. Now $P_{w}$ is a saddle while $P^{*}$ is globally stable. It should be noted that set-points $s^{*}>z^{0}$ violate (18b) all along $\left[z^{0}, s^{*}\right]$. Although $\overline{\mathcal{X}}$ is theoretically not reachable in finite time, practical wash-out may occur for low initial conditions.

Another situation happens when the kinetics exhibits multiplicity as for $\mu_{3}^{*}$. In this case a new fixed point at $P^{u}=\left(1-s_{3}^{u}, s_{3}^{u}, 1\right)$ between $P^{*}$ and $P_{w}$ appears. $P^{u}$ is locally unstable, whereas $P_{w}$ is now stable. This is closely connected with the WAC. In fact, (18b) is violated for all $s>s_{3}^{u}$ and biomass wash-out occurs. As a result, $P^{*}$ is stable but not globally stable. This situation is illustrated with some state trajectories in Fig. 2d.

Many often, continuous bioreactors are operated under high productivity conditions, i.e. the broth is fed with a high substrate concentration $\left(S^{\text {in }}\right)$ flow while dilution $D$ drives $\mu$ close to $\mu_{\max }$ in the range of multiple equi- 
libria. This analysis confirms that stability is critical in chemostats operated under such conditions. In fact, high inhibition is usually present, the domain of attraction of the set-point is constrained to its vicinity and biomass wash-out is prone to occur. That is why, in practice, the dilution is slowly increased from zero to its desired value. Contrarily, in those cases in which productivity is not an issue, globally attractive operating points are possible.

\subsection{Reaction rate proportional control}

Sometimes, the reaction rate $(\mu x)$ can be indirectly known by measuring on-line output gases or other chemical variables [17]. Also, it can be estimated using cell density sensors, that will be briefly described in next subsection. In such cases, a family of controllers of the form $D(x, s)=\gamma(\mu x)$ can be implemented, where $\gamma$ can be constant or adapted along the transient response. Let us consider $\gamma=1 / x^{*}$, with $x^{*}$ being the desired biomass concentration set-point:

$$
D_{0}(x, s)=\frac{1}{x^{*}}(x \mu)
$$

Weak batch and wash-out avoidance conditions become

$$
\begin{array}{ll}
w B A C: \frac{1}{x^{*}}>1 & \forall x \in\left\langle z^{0}, 1\right\rangle \\
W A C: 0<\mu(0, s, \cdot) & \forall s \in\left\langle z^{0}, 1\right\rangle
\end{array}
$$

Clearly, the sBAC (12) is not fulfilled. Then, an initial condition $s^{0}>0$, as it is usual in practice, is thus required. Conversely, the wBAC (21a) is always fulfilled since $x^{*}<1$. Similarly, (21b) is trivially satisfied all along $\overline{\mathcal{X}}$. This is the main advantage of this control structure: both $P_{b}$ and $P_{w}$ are unstable equilibria on $\mathcal{Z}$, whereas there exists a unique stable equilibrium $P^{*}=\left(x^{*}, 1-x^{*}, 1\right)$ for any growth kinetic function. Hence, this controller semiglobally stabilises $P^{*}$ independently of its position on $\mathcal{Z}$.

Replacing $D(t)$ in (4a)-(4b) with (20) yields

$$
\begin{aligned}
\dot{x} & =D_{0}(x, s)\left(x^{*}-x\right) \\
\dot{s} & =D_{0}(x, s)\left(s^{*}-s\right)
\end{aligned}
$$

The concentration dynamics are decoupled one of each other and converge to their steady state values at the same rate, so the orbits starting in $I R^{*}$ are straight lines (see Fig. 3). The main drawback of this controller is that concentrations evolve slowly when any of the concentrations is low because $D$ becomes too low also.

\subsection{Cell density proportional control}

Several sensors have been developed to measure cell concentration on-line. They are commonly based on optical density and cell capacitance measurement $[4,10,22]$. If biomass concentration is measured, a lot of control strategies can be implemented. The simplest one is:

$$
D_{1}(x)=\frac{\mu^{*}}{x^{*}} x
$$

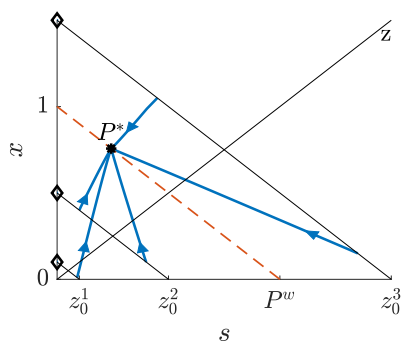

Figure 3: Reaction rate proportional control, $D_{0}$ in eq. (20). State portrait; $*$ : set-point, $\diamond$ : invariant points.

Strong batch and wash-out avoidance conditions become

$$
\begin{array}{ll}
s B A C: \frac{\mu^{*}}{x^{*}} x>0 & \forall x \in\left\langle z^{0}, 1\right\rangle \\
W A C: 0<\mu(0, s, \cdot) & \forall s \in\left\langle z^{0}, 1\right\rangle
\end{array}
$$

It is quite obvious that this controller fulfills both conditions for any $P^{*}$. In contrast with the previous one, $\overline{\mathcal{S}}$ is not invariant, so the control can start from $s^{0}=0$. That is, now $P_{b}$ is not a fixed point, whereas $P_{w}$ is again a saddle. These conditions, however, do not suffice to guarantee $P^{*}$ is globally stable. In fact, substrate inhibition often leads to multiple equilibria. According to (16), the equilibria will be determined by the intersections and eventual tangent points between $\mu(1-s, s, q)$ and line $D_{1}(1-s)=\frac{1-s}{1-s^{*}} \mu^{*}$. There will be a single and globally stable equilibrium $P^{*}$ only in the case of monotonous or slightly inhibited kinetics $\mu(1-s, s, q)$ on $\mathcal{Z}$.

\subsection{Specific growth rate error feedback control}

$D_{1}(x)$ in $(23)$ can be rewritten as $D_{1}(x)=D_{0}(x, s)+$ $\frac{x}{x^{*}}\left(\mu^{*}-\mu\right)$, putting in evidence the implicit feedback of the error in $\mu$. This feedback has a stabilising effect on the $s$-dynamics that makes its convergence to $s^{*}$ faster.

Several specific growth rate observers based on on-line cell density measurement have been developed in the literature and tested experimentally $[1,6]$. Therefore, if $x$ is measured a wide family of controllers feeding back the error in either $\mu$ or $x$ can be designed and implemented.

It is immediate to generalise the specific growth rate error feedback as follows:

$$
D_{\mu 1}(x, s)=D_{0}(x, s)+k \frac{x}{x^{*}}\left(\mu^{*}-\mu\right)
$$

Strong batch and wash-out avoidance conditions become

$$
\begin{array}{ll}
s B A C: k \mu^{*} \frac{x}{x^{*}}>0 & \forall x \in\left\langle z^{0}, 1\right\rangle \\
W A C: 0<\mu(0, s, \cdot) & \forall s \in\left\langle z^{0}, 1\right\rangle
\end{array}
$$

It is quite obvious that (26a)-(26b) hold for any $P^{*}$ and any $k$. Then, global stability is determined by the local 
(a) $D_{\mu 1}(x, s)$ in eq. $(28)$
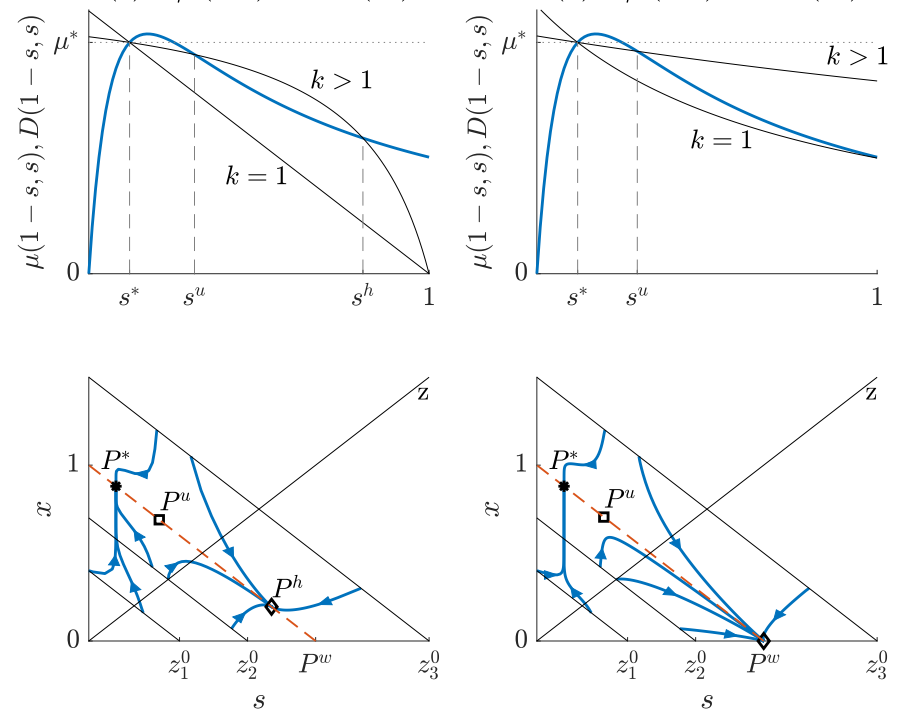

Figure 4: Growth error feedback control (a) eq. (25) and (b) eq. (27). Top: kinetics and equilibria for an arbitrary gain $k$. Bottom: State portrait; *: set-point, $\diamond$ : undesired stable equilibrium, $\square$ : unstable equilibrium.

behaviour on $\mathcal{Z}$. As for (23), multiple equilibria usually appear because of substrate inhibition in $\mu(1-s, s, q)$. Anyway, for monotonic and slightly inhibited kinetics, a single and globally stable equilibrium $P^{*}$ is still possible.

Fig. 4a repeats the kinetics of Fig. 2a. The equilibrium points in $I R^{*} \cap \mathcal{Z}$ are determined by the intersections and eventual tangent points between $\mu(1-s, s, q)$ and $D_{\mu 1}(1-$ $s, s)$ for a given gain $k$. Note that the line labelled with $k=1$ corresponds to control (23) previously discussed. For this particular kinetics, (23) gives a single equilibrium. Instead, control (25) gives three equilibria, two stable $\left(P^{*}\right.$ and $\left.P^{h}\right)$ and one unstable $\left(P^{u}\right)$.

A drawback of feedback law (25) is that the $s$-dynamics is slow when $x^{0}$ is low. To overcome this shortcoming, the proportionality of the feedback term to biomass concentration should be removed. For instance,

$$
D_{\mu 2}(x, s)=D_{0}(x, s)+k\left(\mu^{*}-\mu\right)
$$

Now, the batch and wash-out invariance conditions become

$$
\begin{array}{ll}
s B A C: k \mu^{*}>0 & \forall x \in\left\langle z^{0}, 1\right\rangle \\
W A C: k \mu^{*}<(k+1) \mu(0, s, \cdot) & \forall s \in\left\langle z^{0}, 1\right\rangle
\end{array}
$$

Whereas (28a) holds for any $P^{*}$ and any $k,(28 \mathrm{~b})$ is fulfilled only for small enough $\mu^{*}$. In the limit, as $k \rightarrow \infty$, the WAC (28b) coincides with that of the chemostat (18b).

Fig. $4 \mathrm{~b}$ shows the equilibria resulting from the intersection of $\mu(1-s, s, q)$ with $D_{\mu 2}(1-s, s)$. Two cases are drawn: $k=1$ and $k>1$. Control (27) gives two equilibria for these particular kinetics and feedback gain $k>1$, a stable node $\left(P^{*}\right)$ and an unstable one $\left(P^{u}\right)$. Since $P_{w}$ does not fulfill the WAC (28b), then it is a stable equilibrium that will attract some orbits. This is observed in the state portrait at the bottom of Fig. 4b. This lost of stability is the cost paid by accelerating the response from low $s^{0}$.

Note that specific growth rate error feedback is prone to multiplicity and even instability. The risk of these undesirable behaviours increases with the feedback gain and the set-point. Although neither (25) nor (27) (semi-)globally stabilise the process in the presence of strong inhibition, the population may be lost when using the latter control while the former never washes out the biomass.

\subsection{Turbidostats}

Let us address now the problem of regulating biomass concentration at a given set-point $x^{*}$. Consider

$$
D_{x 1}(x, s)=D_{0}(x, s)+k \frac{\mu^{*}}{x^{*}}\left(x-x^{*}\right)
$$

Strong batch and wash-out avoidance conditions become

$$
\begin{array}{ll}
s B A C: k \frac{\mu^{*}}{x^{*}}\left(x-x^{*}\right)>0 & \forall x \in\left\langle z^{0}, 1\right\rangle \\
W A C:-k \mu^{*}<\mu(0, s, \cdot) & \forall s \in\left\langle z^{0}, 1\right\rangle
\end{array}
$$

The WAC holds trivially for $k>0$. $D_{x 1}(x, 0)>0$ whenever $x^{*}<z^{0}$. That is, $x^{*}$ should be low enough to avoid falling in batch mode from some $P^{0}$. This is the main shortcoming of this feedback strategy. In fact, a set of non-isolated stable equilibria $\left[z^{0}, x^{*}\right]$ on $\overline{\mathcal{S}}$ that will attract some orbits exists whenever $z^{0}<x^{*}$.

Replacing $D(x, s)$ in (16) with (29) yield after some trivial algebra

$$
\frac{x-x^{*}}{x^{*}}\left(\mu(x, 1-x, q)+k \mu^{*}\right)=0
$$

which has a single root $x^{*}$.

So, the process exhibits a single equilibrium $P^{*}=$ $\left(x^{*}, 1-x^{*}, 1\right)$ in $I R^{*} \cap \mathcal{Z}$, while $P_{w}$ is unstable. Summarising, $P^{*}$ is globally stable for those set-points satisfying $x^{*}<z^{0}$, whereas the process falls in batch operation from some initial conditions on $z^{0}<x^{*}$.

Fig. 5a displays the state portrait for control (29). It is seen that the control saturates at zero in all trajectories starting at $x^{0}<<x^{*}$. Saturation is transitory for the initial conditions on $z_{2}^{0}$ and $z_{3}^{0}$ but is permanent for an initial condition on $z_{1}^{0}$.

Keeping in mind (30a), the feedback law can be easily redesigned to guarantee global convergence for any setpoint including $x^{*}>z^{0}$. For instance, if the dilution rate is computed as follows

$$
D_{x 2}(x, s)=D_{0}(x, s)+\frac{k}{\mu^{*} x^{*}} \mu^{2}\left(x-x^{*}\right)
$$

then

$$
\begin{array}{ll}
w B A C: \frac{1}{x^{*}}>1 & \forall x \in\left\langle z^{0}, 1\right\rangle \\
W A C:-k \mu(0, s, \cdot)<\mu^{*} & \forall s \in\left\langle z^{0}, 1\right\rangle
\end{array}
$$


(a) $D_{x 1}(x, s)$ in eq. (33)

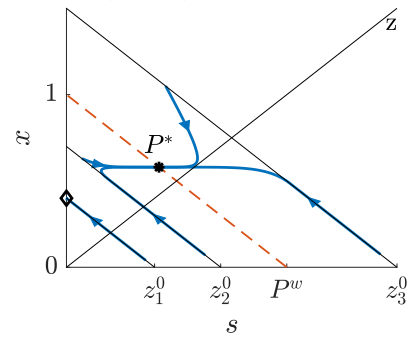

(b) $D_{x 2}(x, s)$ in eq. (36)

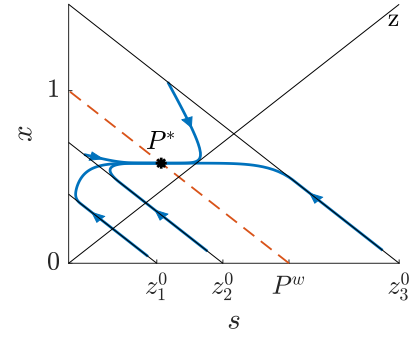

Figure 5: Turbidostat, (a) eq. (29) and (b) eq. (32). State portraits; $*$ : set-point, $\diamond$ : undesired stable equilibrium.

Now, an initial condition $s^{0}>0$ is required. However, (33a)-(33b) are trivially satisfied for any $P^{*}$, independently of its position on $\mathcal{Z}$. Therefore, both $P_{b}$ and $P_{w}$ are unstable equilibria on $\mathcal{Z}$. Like before, it can be checked that $D_{x 2}(x, 1-x)$ gives a single root for $(16)$, so there exists a unique stable equilibrium point $P^{*}$. Hence, this controller semi-globally stabilises any $P^{*}$.

Fig. 5b depicts the state portrait for control (32) from the same initial conditions as in Fig. 5a. It is seen that the control saturates at zero in all trajectories starting at $x^{0}<<x^{*}$ but saturation is transitory in all cases. In fact, all state trajectories finally converge to $P^{*}$.

\subsection{Nutristats}

In some bioprocesses, substrate concentration can be reliably measured on-line [15, 25]. Therefore, the process can be driven to a substrate concentration set-point by feeding back the substrate concentration error. Like turbidostats, nutristats have the advantage that a unique equilibrium on $I R^{*} \cap \mathcal{Z}$ is achieved even in the presence of inhibition.

Note that substrate concentration measurement allows estimating the substrate consumption rate $(\mu x)$ by means of observers [12]. So, controllers similar to (25) and (27) can also be implemented. Consider, as starting point, a substrate concentration error feedback strategy of the form

$$
D_{s 1}(x, s)=D_{0}(x, s)+k \frac{\mu^{*}}{s^{*}}\left(s^{*}-s\right)
$$

Strong batch and wash-out avoidance conditions become

$$
\begin{array}{ll}
s B A C: k \mu^{*}>0 & \forall x \in\left\langle z^{0}, 1\right\rangle \\
W A C: k \mu^{*}\left(1-\frac{s}{s^{*}}\right)<\mu(0, s, \cdot) & \forall s \in\left\langle z^{0}, 1\right\rangle
\end{array}
$$

The sBAC holds trivially for any $k>0$ and any set-point $s^{*}$. Instead, the WAC is trivially satisfied whenever $z^{0}>$ $s^{*}$. Otherwise, it is satisfied only for a set of set-points determined by $k$ and the kinetics. Replacing $D(x, s)$ in (16) with (34) and solving for $s$ yield

$$
\left(s-s^{*}\right)\left(\frac{\mu(x, 1-x, q)}{1-s^{*}}+k \frac{\mu^{*}}{s^{*}}\right)=0
$$

(a) $D_{s 1}(x, s)$ in eq. (39)

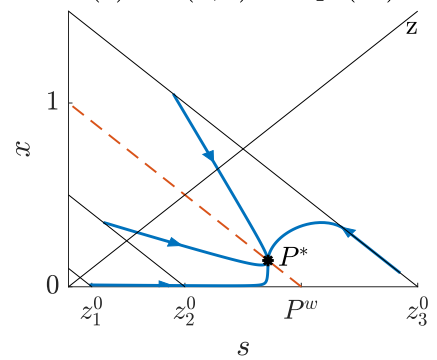

(b) $D_{s 2}(x, s)$ in eq. (42)

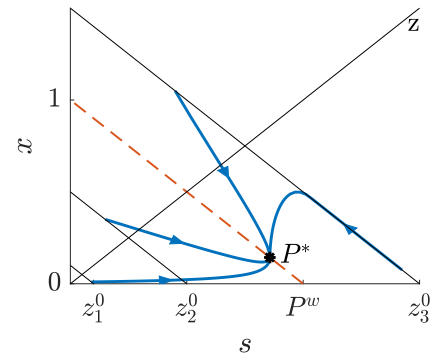

Figure 6: Substrate concentration error feedback control (a) eq. (34) and (b) eq. (37) (Nutristat). State portraits; o: initial state, $*$ : setpoint.

It is obvious from (36) that the process exhibits a single equilibrium $P^{*}=\left(1-s^{*}, s^{*}, 1\right)$ in $\mathcal{Z}$, besides $P_{w}$. Note also that $P_{w}$ is always unstable and $P^{*}$ is always stable.

Fig. 6a shows a state portrait for control (34). Global convergence to $P^{*}$ is observed. However, the risk of biomass wash-out is clear. Particularly, observe how the trajectory starting on $z_{1}^{0}<<s^{*}$ approaches $\overline{\mathcal{X}}$.

The fedback law can be redesigned to fulfill the WAC for any set-point $s^{*}$. For instance, if $D$ is

$$
D_{s 2}(x, s)=D_{0}(x, s)\left(1+k \frac{1}{s^{*}}\left(s^{*}-s\right)\right)
$$

then

$$
\begin{array}{cc}
w B A C: \frac{1}{x^{*}}(1+k)>1 & \forall x \in\left\langle z^{0}, 1\right\rangle \\
W A C: 0<\mu(0, s, \cdot) & \forall s \in\left\langle z^{0}, 1\right\rangle
\end{array}
$$

Now, an initial condition $s^{0}>0$ is required. On the other hand, conditions (38a)-(38b) are trivially satisfied for any $P^{*}$, independently of its position on $\mathcal{Z}$. Therefore, both $P_{b}$ and $P_{w}$ are unstable equilibria on $\mathcal{Z}$. The uniqueness of solution of (16) can be checked as before, so there exists only one stable equilibrium point $P^{*}$. Hence, this controller semi-globally stabilises any $P^{*}$.

Fig. $6 \mathrm{~b}$ depicts the state portrait for control (37) from the same initial conditions as in Fig. 6a. It is seen now that the risk of biomass wash-out from initial conditions on $z^{0}<<s^{*}$ is eliminated.

\section{Discussion}

Table 1 is a summary of the control laws analysed in the paper. The feedback laws written in terms of the original concentrations are found in the first column. (Semi-)global stability (GS), strong or weak batch avoidance condition (s/wBAC) and wash-out avoidance condition (WAC) fulfillment are indicated in the remaining columns. Symbol $\checkmark(\times)$ should be read "holds for any" (respectively, "fails for some") kinetics, set-point and/or initial condition. 


\begin{tabular}{|c|c|c|c|c|}
\hline \multicolumn{2}{|c|}{ Dilution } & \multirow{2}{*}{$\frac{\text { GS }}{\times}$} & \multirow{2}{*}{$\frac{(\mathrm{s} / \mathrm{w}) \mathrm{BAC}}{\mathrm{s} \checkmark}$} & \multirow{2}{*}{$\frac{\text { WAC }}{x}$} \\
\hline Chemostat & $D^{*}=\mu^{*}$ & & & \\
\hline Reaction rate proportional & $D_{0}=\mu \frac{X}{X^{*}}$ & $\checkmark$ & $\mathrm{w} \checkmark$ & $\checkmark$ \\
\hline Cell density proportional & $D_{1}=\mu^{*} \frac{X}{X^{*}}$ & $\times$ & s $\checkmark$ & $\checkmark$ \\
\hline \multirow[t]{2}{*}{ Specific growth rate error feedback } & $D_{\mu 1}=\mu \frac{X}{X^{*}}+k \frac{X}{X^{*}}\left(\mu^{*}-\mu\right)$ & $\times$ & s $\checkmark$ & $\checkmark$ \\
\hline & $D_{\mu 2}=\mu \frac{X}{X^{*}}+k\left(\mu^{*}-\mu\right)$ & $\times$ & s $\checkmark$ & $x$ \\
\hline \multirow[t]{2}{*}{ Turbidostat } & $D_{x 1}=\mu \frac{X}{X^{*}}+k \frac{\mu^{*}}{X^{*}}\left(X-X^{*}\right)$ & $\checkmark$ & $x$ & $\checkmark$ \\
\hline & $D_{x 2}=\mu \frac{X}{X^{*}}+k \frac{\mu^{2}}{\mu^{*} X^{*}}\left(X-X^{*}\right)$ & $\checkmark$ & $\mathrm{w} \checkmark$ & $\checkmark$ \\
\hline \multirow[t]{2}{*}{ Nutristat } & $D_{s 1}=\frac{(y \mu X)}{S_{j}^{i n}-S^{*}}+k \frac{\mu^{*}}{S^{*}}\left(S^{*}-S\right)$ & $\checkmark$ & s $\checkmark$ & $\times$ \\
\hline & $D_{s 2}=\frac{(y \mu X)}{S_{j}^{i n}-S^{*}}\left(1+\frac{k}{S^{*}}\left(S^{*}-S\right)\right)$ & $\checkmark$ & $\mathrm{w} \checkmark$ & $\checkmark$ \\
\hline
\end{tabular}

Table 1: Summary of feeding laws and properties.

Application to other control laws. The feedback laws treated in this paper are designed using $D_{0}:=\frac{\mu X}{X^{*}}=$ $\frac{y \mu X}{S^{i n}-S^{*}}$ as a basis. However, any other type of continuous feedback satisfying assumptions 2.3-3.1 can be designed or analysed. For instance, the tools developed here can be used to reshape feedback linearisation control laws in the vicinities of $\overline{\mathcal{X}}$ and $\overline{\mathcal{S}}$ in order to avoid biomass washout and batch mode operation, respectively.

Application in adaptive control. The main source of uncertainty in the mass balance model is the kinetics. This uncertainty can produce appreciable state errors when the feedback law requires complete characterisation of the operating point (for instance, $X^{*}$ and $\mu^{*}$ ). The yield $y$ is another source of error because it usually varies with time and with the operating point. Also, the inlet substrate concentration $S^{\text {in }}$ is not always well characterised. They are well known the difficulties to globally stabilise bioreactors by means of PI-like controllers [7]. Adaptive control, where some controller gain is adapted between certain limits using integral action, is an attractive option [17]. The results presented here are also useful to shape the adaptation law, particularly the limits of the adaptation span. For instance, recall the control $D(x, s)=\gamma(\mu x)$ of section 4.2. Suppose $\gamma$ is continuously adapted instead of being constant and equal to $1 / x^{*}$. It is immediate from (21a) that a lower limit $\gamma>1$ is required to fulfill the BAC and therefore required to semi-globally stabilise the reactor.

Extension to other kinetic models. Some kinetic models do not hold Assumption 2.1. For instance, there are kinetic functions satisfying $\mu(\cdot, S, \cdot)=0 \forall S>S^{m}$ that fit well for very toxic substrates when in excess [13]. Biomass is washed out if the control drives the process to such high substrate concentration levels, so the problem is crit- ical when $S^{i n}(t)>S^{m}$. To avoid biomass wash-out, the control should verify an additional condition, which essentially consists in the non-attractiveness of the set $\mathcal{S}^{m}=\left\{(x, s, z) \in I R_{0} \mid s=s^{m}\right\}$ from the left:

$$
\lim _{s \rightarrow s^{m}-} \frac{D(x, s)}{\mu(x, s, \cdot)}\left(1-s^{m}\right)<x \quad \forall x \in\left\langle z^{0}-s^{m}, 1-s^{m}\right\rangle
$$

Extension to other types of bioprocesses. The results of this paper can be also applied to more complex bioreactions. For instance, consider a dual substrate bioreaction with additive kinetics. Its mass balance model can be written after suitable normalisation as

$$
\begin{aligned}
\dot{x} & =\mu\left(x, s_{1}, s_{2}\right) x-D\left(x, s_{1}, s_{2}\right) x \\
\dot{s_{1}} & =-\mu_{1}\left(x, s_{1}\right) x-D\left(x, s_{1}, s_{2}\right) s_{1}+a_{1} D\left(x, s_{1}, s_{2}\right) \\
\dot{s_{2}} & =-\mu_{2}\left(x, s_{2}\right) x-D\left(x, s_{1}, s_{2}\right) s_{2}+a_{2} D\left(x, s_{1}, s_{2}\right) \\
\dot{z} & =D\left(x, s_{1}, s_{2}\right)(1-z)
\end{aligned}
$$

with $\mu\left(x, s_{1}, s_{2}\right)=\mu_{1}\left(x, s_{1}\right)+\mu_{2}\left(x, s_{2}\right), a_{1}+a_{2}=1$ and $z=x+s_{1}+s_{2}$.

The extension of the washout avoidance condition (8a) to the dual substrate case is straightforward:

$$
\lim _{x \rightarrow 0^{+}} D\left(x, s_{1}, s_{2}\right)<\mu\left(x, s_{1}, s_{2}\right) \quad \forall s_{1}+s_{2} \in\left\langle z^{0}, 1\right\rangle
$$

The strong (12) and weak (13) batch avoidance conditions can also be extended to the dual substrate case:

$$
\begin{gathered}
D(x, 0,0)>0 \forall x \in\left\langle z^{0}, 1\right\rangle \\
\lim _{s_{1}, s_{2} \rightarrow 0^{+}} \frac{D\left(x, s_{1}, s_{2}\right)}{\mu\left(x, s_{1}, s_{2}\right)}>x \quad \forall x \in\left\langle z^{0}, 1\right\rangle
\end{gathered}
$$

Based on these results, the (semi-)global stabilisation of dual substrate continuous processes can be investigated 
with a similar procedure as before. Additional conditions should be established to avoid biomass growing on only one of the substrates instead of on both of them. Further, the dynamics on the reaction invariant $z=1$ is now of order two, so more complex behaviour may appear.

\section{Conclusions}

It was proved that global stability features of the bioreaction (4) can be determined by checking the invariance of the strictly positive quadrant in the state space and finding the roots of a nonlinear equation. This approach offers mathematical tools to understand and re-think trade-offs and limitations of empirical control laws so common in biotechnology. Also, and more importantly, it provides a simple and systematic procedure to design and redesign globally stabilising control laws without the need of searching for Lyapunov functions and domains of attraction.

A distinctive property of the proposed approach is that feedback law saturation does not modify the stability results. In other words, if the invariant conditions on the border of the operating region are fulfilled, then stability is guaranteed no matter if the dilution saturates or not.

Although the proposed approach was developed for state feedback control where a pure culture grows on a single substrate, the results can be extended to more general bioreactions and controllers. In fact, the invariant conditions can be exploited to shape the adaptation law in adaptive control. Also, the control design procedure can be extended to stabilise more complex bioreactions such those involving two substrates with additive dynamics. The development of similar design tools for fixed-bed [17] and perfusion [9] bioprocesses in which the dynamic restriction (2) does not hold is also an interesting open problem.

\section{Acknowledgements}

Financed by I216-2016 (UNLP), PICT2014-2394 (ANPCyT) and PIP112-2015-01-00837 (CONICET), Argentina; and by DPI2014-55276-C5-1-R (MCI), Spain.

\section{References}

[1] G. Bastin and D. Dochain. On-line estimation of microbial specific growth rates. Automatica, 22:705-709, 1986.

[2] G. Bastin and J. Van Impe. Nonlinear and adaptive control in biotechnology: A tutorial. European J. Control, 1:37-53, 1995.

[3] K. Bellgardt. Bioreaction Engineering, Ch.: Bioprocess Model, pages 44-105. Springer, 2000

[4] A. Bolic, H. Larsson, S. Hugelier, An. Lantz, U. Krahne, and K. Gernaey. A flexible well-mixed milliliter-scale reactor with high oxygen transfer rate for microbial cultivations. Chemical Engineering J., 303:655 - 666, 2016.

[5] B. Canton, A. Labno, and D. Endy. Refinement and standardization of synthetic biological parts and devices. Nature Biotechnology, 26:787-793, 2008.
[6] H. De Battista, J. Picó, F. Garelli, and A. Vignoni. Specific growth rate estimation in (fed-)batch bioreactors using secondorder sliding observers. J. Process Control, 21:1049-1055, 2011.

[7] H. De Battista, J. Picó, and E. Picó-Marco. Nonlinear PI control of fed-batch processes for growth rate regulation. J. Process Control, 22:789 - 797, 2012

[8] J. de Vree, Rouke Bosma, R. Wieggers, S. Gegic, M. Janssen, M. Barbosa, and R. Wijffels. Turbidostat operation of outdoor pilot-scale photobioreactors. Algal Research, 18:198 - 208, 2016.

[9] J. Deschênes, A. Desbiens, M. Perrier, and A. Kamen. Multivariable nonlinear control of biomass and metabolite concentrations in a high-cell-density perfusion bioreactor. Industrial \& Engineering Chemistry Research, 45:8985-8997, 2006.

[10] B. Downey, L. Graham, J. Breit, and N. Glutting. A novel approach for using dielectric spectroscopy to predict viable cell volume (VCV) in early process development. Biotechnology Progress, 30:479-487, 2014.

[11] I. Dunn, E. Heinzle, J. Ingham, and J. Přenosil. Biological Reaction Engineering. Dynamic Modelling Fundamentals with Simulation Examples. Wiley-VCH Verlag, 2003.

[12] M. Farza, K. Busawon, and H. Hammouri. Simple nonlinear observers for on-line estimation of kinetic rates in bioreactors. Automatica, 34:301-318, 1998.

[13] Y. Hwang, D. Dochain, and G. Bastin. Performance of inhibition functions with total inhibition concentration. In IFAC Computer Applications in Biotechnology, 1995.

[14] I. Karafyllis, M. Malisoff, and M. Krstic. Sampled-data feedback stabilization of age-structured chemostat models. In American Control Conf., 2015.

[15] G. Kleman, J. Chalmers, G. Luli, and W. Strohl. Glucose-stat, a glucose-controlled continuous culture. Applied and Environmental Microbiology, 57:918-923, 1991.

[16] K. Lee, P. Boccazzi, A. Sinskey, and R. Ram. Microfluidic chemostat and turbidostat with flow rate, oxygen, and temperature control for dynamic continuous culture. Lab Chip, 11:1730-1739, 2011.

[17] L. Mailleret, O. Bernard, and J. Steyer. Nonlinear adaptive control for bioreactors with unknown kinetics. Automatica, 40:1379 $-1385,2004$.

[18] F. Mazenc, J. Harmand, and M. Malisoff. Stabilization in a chemostat with sampled and delayed measurements. In American Control Conf., 2016.

[19] A. Nakano, C. Lee, A. Yoshida, T. Matsumoto, N. Shiomi, and S. Katoh. Effects of methanol feeding methods on chimeric alpha-amylase expression in continuous culture of pichia pastoris. J. Bioscience and Bioengineering, 101:227 - 231, 2006.

[20] T. Paalme, R. Elken, A. Kahru, K. Vanatalu, and R. Vilu. The growth rate control in Escherichia coli at near to maximum growth rates: the A-stat approach. Antonie van Leeuwenhoek, 71:217-230, 1997.

[21] M. Perrier and D. Dochain. Evaluation of control strategies for anaerobic digestion processes. International J. Adaptive Control and Signal Processing, 7:309-321, 1993.

[22] E. Picó-Marco, J. Picó, and H. De Battista. Sliding mode scheme for adaptive specific growth rate control in biotechnological fed-batch processes. Int. J. Control, 78:128-141, 2005.

[23] T. Proll and N. Karim. Nonlinear control of a bioreactor model using exact and I/O linearization. Int. J. Control, 60:499-519, 1994.

[24] A. Rapaport and J. Harmand. Robust regulation of a class of partially observed nonlinear continuous bioreactors. J. Process Control, 12:291 - 302, 2002.

[25] M. Rutgers, A. Breure, and J. van Andel. Nutristat: a substrate concentration-controlled continuous culture as a tool to grow bacteria on toxic compounds. In Institution of Chemical Engineers Symposium Series, pages 161-163, 1994.

[26] A. Saldanha, M. Brauer, and D. Botstein. Nutritional homeostasis in batch and steady-state culture of yeast. Molecular Biology of the Cell, 15:4089-4104, 2014.

[27] M. Sbarciog, M. Loccufier, and E. Noldus. Convergence and stability of biochemical reaction systems of rank one. In IEEE 
Conf. Decision and Control, 2005.

[28] M. Scott, C. Gunderson, E. Mateescu, Z. Zhang, and T. Hwa. Interdependence of cell growth and gene expression: Origins and consequences. Science, 330:1099-1102, 2010.

[29] E. Simova, D. Beshkova, M. Angelov, and Z. Dimitrov. Bacteriocin production by strain lactobacillus delbrueckii ssp. bulgaricus bb18 during continuous prefermentation of yogurt starter culture and subsequent batch coagulation of milk. J. Industrial Microbiology \& Biotechnology, 35:559-567, 2008.

[30] K. Stamatelatou, L. Syrou, C. Kravaris, and G. Lyberatos. An invariant manifold approach for CSTR model reduction in the presence of multi-step biochemical reaction schemes. Application to anaerobic digestion. Chemical Engineering J., 150:462 $-475,2009$

[31] C. Takahashi, A. Miller, F. Ekness, M. Dunham, and E. Klavins. A low cost, customizable turbidostat for use in synthetic circuit characterization. ACS Synthetic Biology, 4:32-38, 2015. 